\title{
Novel Augmentation Technique for Patellar Tendon Repair Improves Strength and Decreases Gap Formation: A Cadaveric Study
}

\author{
James C. Black MD, William M. Ricci MD, Michael J. Gardner MD, \\ Christopher M. McAndrew MD, MSc, Avinesh Agarwalla BS, \\ Robert D. Wojahn MD, Orchid Abar, Simon Y. Tang PhD
}

Received: 11 March 2016/Accepted: 27 July 2016/Published online: 4 August 2016

(C) The Association of Bone and Joint Surgeons(B) 2016

\begin{abstract}
Background Patellar tendon ruptures commonly are repaired using transosseous patellar drill tunnels with modified-Krackow sutures in the patellar tendon. This simple suture technique has been associated with failure rates and poor clinical outcomes in a modest proportion of patients. Failure of this repair technique can result from gap formation during loading or a single catastrophic event. Several augmentation techniques have been described to improve the integrity of the repair, but standardized biomechanical evaluation of repair strength among different techniques is lacking.

Questions/purposes The purpose of this study was to describe a novel figure-of-eight suture technique to
\end{abstract}

The institution of one of the authors (JCB) has received, during the study period, funding from the AOTrauma North America. One of the authors certifies that he (JCB), has received payments, during the study period, an amount of USD 10,000-USD 100,000 from the AOTrauma North America (Paoli, PA, USA).

All ICMJE Conflict of Interest Forms for authors and Clinical Orthopaedics and Related Research ${ }^{\circledR}$ editors and board members are on file with the publication and can be viewed on request.

This study was performed at Washington University School of Medicine, St Louis, MO, USA.

J. C. Black ( $\square)$

Mercy Orthopedic Trauma, Mercy Springfield, 3050 E.

Riverbluff Blvd, Ozark, MO 65721, USA

e-mail: blackjamesc@gmail.com

W. M. Ricci, C. M. McAndrew, A. Agarwalla, R. D. Wojahn, O. Abar, S. Y. Tang

Department of Orthopaedic Surgery, Washington University

School of Medicine, St Louis, MO, USA

M. J. Gardner

Department of Orthopaedic Surgery, Stanford University School of Medicine, Redwood City, CA, USA augment traditional fixation and evaluate its biomechanical performance. We hypothesized that the augmentation technique would (1) reduce gap formation during cyclic loading and (2) increase the maximum load to failure.

Methods Ten pairs (two male, eight female) of freshfrozen cadaveric knees free of overt disorders or patellar tendon damage were used (average donor age, 76 years; range, 65-87 years). For each pair, one specimen underwent the standard transosseous tunnel suture repair with a modified-Krackow suture technique and the second underwent the standard repair with our experimental augmentation method. Nine pairs were suitable for testing. Each specimen underwent cyclic loading while continuously measuring gap formation across the repair. At the completion of cyclic loading, load to failure testing was performed.

Results A difference in gap formation and mean load to failure was seen in favor of the augmentation technique. At 250 cycles, a $68 \%$ increase in gap formation was seen for the control group (control: $5.96 \pm 0.86 \mathrm{~mm}$ [95\% CI, 5.30$6.62 \mathrm{~mm}$ ]; augmentation: $3.55 \pm 0.56 \mathrm{~mm}$ [95\% CI, 3.12$3.98 \mathrm{~mm}] ; \mathrm{p}=0.02$ ). The mean load to failure was $13 \%$ greater in the augmentation group (control: $899.57 \pm 96.94$ $\mathrm{N}$ [95\% CI, 825.06-974.09 N]; augmentation: $1030.70 \pm$ $122.41 \mathrm{~N}$ [95\% CI, 936.61-1124.79 N]; p = 0.01).

Conclusions This biomechanical study showed improved performance of a novel augmentation technique compared with the standard repair, in terms of reduced gap formation during cyclic loading and increased maximum load to failure.

Clinical Relevance Decreased gap formation and higher load to failure may improve healing potential and minimize failure risk. This study shows a potential biomechanical advantage of the augmentation technique, providing support for future clinical investigations comparing this 
technique with other repair methods that are in common use such as transosseous suture repair.

\section{Introduction}

Rupture of the patellar tendon is a potentially disabling injury typically often treated with surgical repair [11, 20, 25-28, 37]. Successful repair can prove challenging owing to the high mechanical loads across the repair and the impaired healing potential of tendons because of their relatively poor blood supply. Incidence of patellar tendon injuries is estimated at 0.68 of 100,000 persons per year, with a mean age at rupture of 49 years for males and 69 years for females [4]. Medical comorbidities such as diabetes, renal failure, systemic lupus erythematosus, and long-term corticosteroid therapy have been implicated in an increased risk of tendon rupture [12, 14, 29]. Pathologic examination of ruptured tendons has shown histopathologic changes of degenerative tendinopathy, chronic tendinitis, and collagen degradation, which is particularly true for patients who sustain bilateral ruptures [39].

Surgical treatment of patellar tendon ruptures is indicated in patients fit for elective surgery to restore integrity of the extensor mechanism; the most-commonly described technique involves four-strand suture fixation of the tendon passed through transosseous patellar tunnels $[11,20,27,32]$. The repair is tensioned and the sutures tied over the superior pole of the patella. Use of this simple suture technique has been associated with failure rates requiring reoperation as high as $66 \%$ in rupture after TKA $[2,17,34]$, but no failure data have been clearly reported for suture repair of acute ruptures of native knees. The feared complication of catastrophic repair failure historically has led many surgeons to recommended a period of postoperative knee immobilization in extension to decrease the strain across the repair [13, 20, 22, 30, 33, 37]; however, the benefits of early motion have been shown to include adhesion reduction, improved knee motion, and promotion of tendon healing with earlier remodeling of collagen fibers and increased collagen filaments crosslinking and strength [8-10, 21, 24, 31, 38]. As a result, several augmentation techniques have been described to allow for earlier motion and full weightbearing and perhaps decreasing the risk of failure $[1,6,13,15,16,23,25,26$, 28, 30, 33, 36, 40]. Many of the proposed augmentation techniques have required a second operation for removal of the hardware or synthetic material used in the augmentation $[6,15,26,36]$. Despite numerous small retrospective studies that exist describing patellar tendon repairs with and without augmentation, standardized evaluation of the repairs is lacking and no clinical trials exist comparing different repair methods. Only more recently have biomechanical studies appeared which systematically compare the strength and efficacy of various techniques, but the preferred repair method often is debated [3, 7, 19, 35]. Additionally, the role of gap formation across a repair and the loads experienced by the repair before catastrophic failure are not well defined. A better appreciation of these variables could result in more resilient repairs and improved outcomes.

The purpose of our study was to describe and evaluate the mechanical properties of a novel patellar tendon suture repair technique, which was developed and used clinically at our institution with promising anecdotal clinical results. The described technique is a simple augmentation to standard transosseous tunnel suture repair. We hypothesized that the novel augmentation technique would (1) reduce gap formation during cyclic loading and (2) increase the maximum load to failure compared with standard suture repair without augmentation.

\section{Methods}

A biomechanical testing model was developed based on work described by Ravalin et al. [35]. Twenty fresh-frozen cadaveric knees, 10 left and right pairs, harvested midthigh to mid-tibia, were used for comparison between the two repair techniques. The average donor age was 76 years (range, 65-87 years), with two males and eight females. The specimens were free of any overt pathologic conditions and the patellar tendons were inspected for any preexisting damage. The skin and subcutaneous tissue were removed, preserving the extensor mechanism, retinaculum, and joint capsule. To simulate a patellar tendon rupture at the level of the inferior pole of the patella, each specimen tendon was transversely cut $2 \mathrm{~mm}$ distal to the inferior pole. The specimen pairs were blindly randomized to one of two groups: (1) left knee control/right knee augmentation or (2) right knee control/left knee augmentation; allocating five pairs in each group. Given their randomization, one knee was repaired using the standard transosseous tunnel suture approach with a modifiedKrackow suture technique (control) and the other knee received the experimental augmentation repair technique. The same surgeon (JCB) performed all repairs to standardize technique and minimize confounding factors and variability between repair types.

\section{Control Repair Technique}

Two Number 5 Ethibond $^{\mathrm{TM}}$ (Ethicon Inc, Somerville NJ, USA) sutures were woven into the patellar tendon using the 
suture technique described by Krackow et al. [18]. Three vertical transosseous bone tunnels were made in the patella using a $2.5-\mathrm{mm}$ drill bit. The two central suture limbs from each Krackow loop were passed through the central bone tunnel and the remaining medial and lateral suture limbs were passed through their respective medial and lateral bone tunnels. Using a free needle, the two central sutures were passed through the quadriceps tendon along the superior pole of the patella to the corresponding medial and lateral sutures to minimize tissue entrapment with knot tying. The repair was tensioned and the sutures tied with the knee in $30^{\circ}$ flexion (Fig. 1).

\section{Experimental Repair Technique}

The suture repair via three vertical transosseous bone tunnels was performed as described for the control technique. Augmentation used two additional oblique drill tunnels made between the vertical tunnels; each oblique drill tunnel was made with a $2.5-\mathrm{mm}$ drill bit, beginning at the inferior pole of the patella and exiting on the anterior patellar surface. One Number 5 Ethibond $^{\mathrm{TM}}$ suture was placed in a figure-of-eight fashion through these two oblique patellar bone tunnels to capture the proximal extent of the modified-Krackow suture repair (Fig. 2). Specifically, the augmentation suture is passed through one

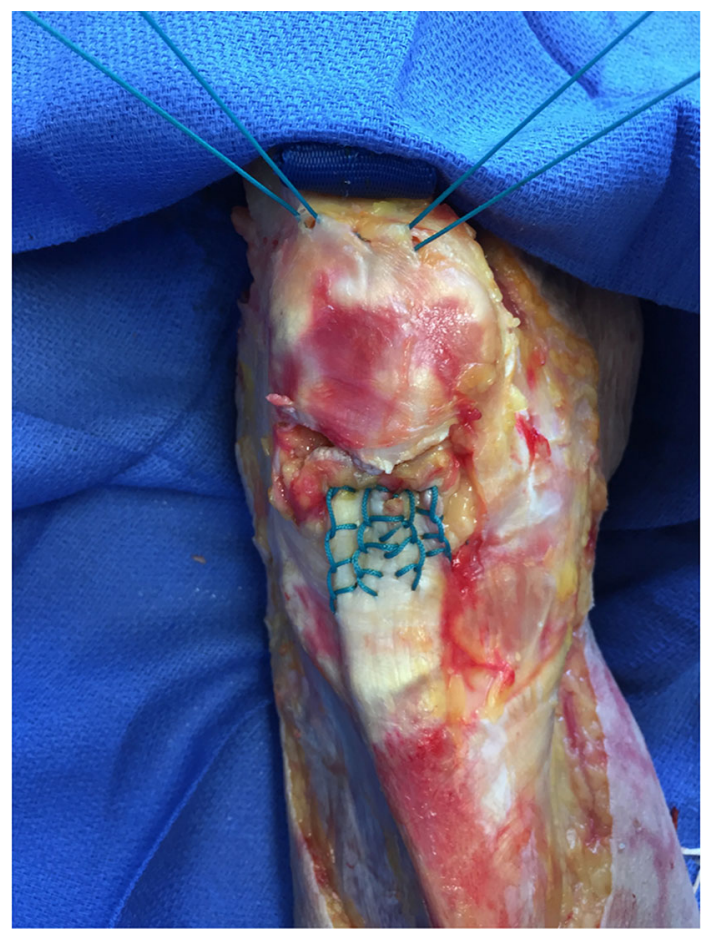

Fig. 1 The modified-Krackow stitch and transosseous tunnel repair technique is shown before final tying of the suture over the superior pole of the patella. oblique tunnel from proximal to distal, then through the ruptured end of the tendon, posterior to anterior (capturing the Krackow loops), then through the other oblique tunnel, and then again through the ruptured end of the tendon, posterior to anterior (capturing the Krackow loops). This allows the augmentation suture to tension the entire modified-Krackow repair to the patella without pulling out through the tendon. With the knee in $30^{\circ}$ flexion, all sutures were tensioned and the central figure-of-eight augmentation suture was tied first followed by the sutures along the superior patella (Fig. 3).

\section{Testing Apparatus and Protocol}

The study began with 10 pairs of specimen knees, but one pair was excluded owing to technical failure of the motion capture system to accurately record gap data, leaving nine pairs of knees available for analysis. For each tested specimen, 5-mm diameter spherical reflective markers were placed on both sides of the repair and affixed using cyanoacrylate glue; one marker secured to the anterior patellar surface, a second marker distally on the patellar tendon, and a third marker on the tibial tubercle (Fig. 4). The three reflective markers allowed for real-time tracking of the gap formation across the repair site using a Qualisys (Highland Park, IL, USA) motion capture system with two tracking cameras at a sampling rate of $1000 \mathrm{~Hz}$. This system can resolve spatial differences down to $0.1 \mathrm{~mm}$. Systematic translation and rotation of the individual markers that occurs during testing is accounted for by the processing software and gap formation is calculated only on the relative motion between markers.

The quadriceps tendon was sutured to 1-inch nylon webbing with a minimum of 10 interrupted Number 5 Ethibond $^{\mathrm{TM}}$ mattress sutures. A proximal loop in the nylon webbing allowed for connection to an Instron ${ }^{\circledR}$ Model 5866 servohydraulic testing machine (Instron ${ }^{\circledR}$, Norwood, MA, USA) equipped with a $10-\mathrm{kN}$ load cell via a cable and pulley mechanism. The femur was secured to a custom apparatus allowing the tibia to hang free with the knee at $90^{\circ}$ at rest (Fig. 5). To simulate the weight of an intact leg and foot, a 5-pound weight was attached to the tibia via external fixation components $33 \mathrm{~cm}$ distal to the medial femoral epicondyle (axis of rotation). Previous studies have used this construct, which simulates the moment arm of an average $70-\mathrm{kg}$ person (Fig. 6) [3, 5, 19, 35].

Using displacement control parameters, each specimen then was extended to $5^{\circ}$ for 250 cycles at $1 \mathrm{~mm}$ per second whereby each specimen was extended to $5^{\circ}$ from the $90^{\circ}$ position. From there, the limb was returned to $90^{\circ}$ by gravity under position control. No phase lag was observed as the limb was not allowed to enter the next extension 
Fig. 2A-C (A) The modifiedKrackow suture repair technique is shown with two additional inferior to anterior oblique drill tunnels and (B) placement of a figure-of-eight augmentation stitch capturing the Krackow loops. (C) The repair is tensioned and the figure-of-eight stitch is tied first, followed by the Krackow tag ends over the superior pole of the patella. (Published with permission from Scott Bodell, Bodell Communications Inc Dallas, TX, USA.)
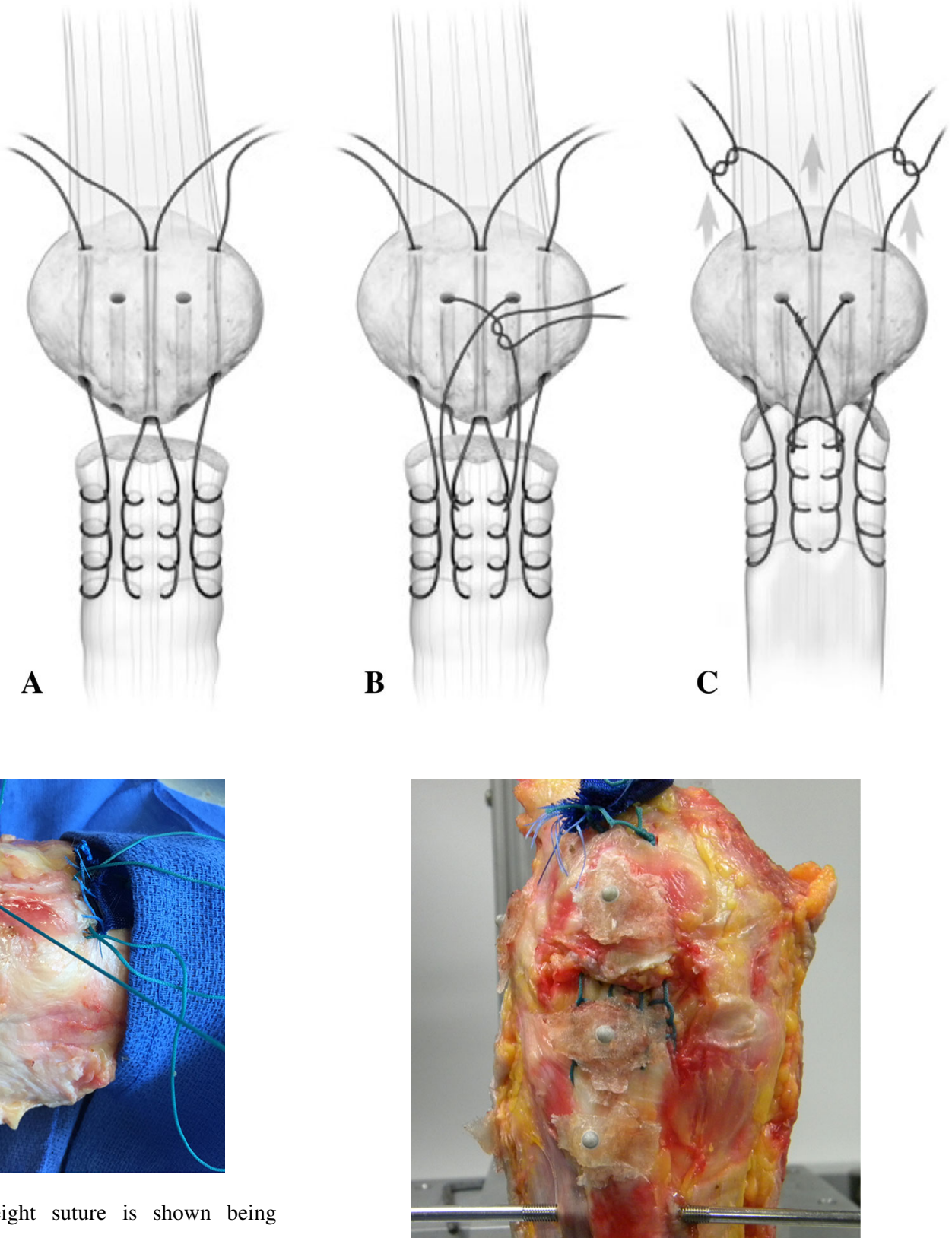

Fig. 3 The augmentation figure-of-eight suture is shown being tensioned and tied first.

cycle until it had flexed to the original $90^{\circ}$ position. The flexion angle was determined dynamically using the Qualisys motion capture system. The force applied across the repair site was recorded through each cycle and the motion capture system continuously measured gap formation across the repair site. As described by Ravalin et al. [35], 250 cycles was chosen as the primary endpoint for cyclic evaluation. Gap formation was recorded for analysis after $1,10,25,50,100,150,200$, and 250 cycles during cyclic load testing. At the completion of 250 cycles, the knee was fixed in $45^{\circ}$ flexion and increasing axial loads were applied at a fixed displacement of $1 \mathrm{~mm}$ per second until the repair construct failed. We used this failure testing
Fig. 4 Three reflective markers were placed to capture gap formation across the repair site using the Qualisys motion tracking system.

model as described by Bushnell et al. [3]. The mode of failure and applied force were recorded at catastrophic failure. The specimens were kept hydrated with normal saline spray throughout testing.

\section{Statistical Methods}

Two-way ANOVA testing (with Tukey's post hoc multiple comparisons) was performed for the gap formation data where cycle number and repair techniques are assumed to 


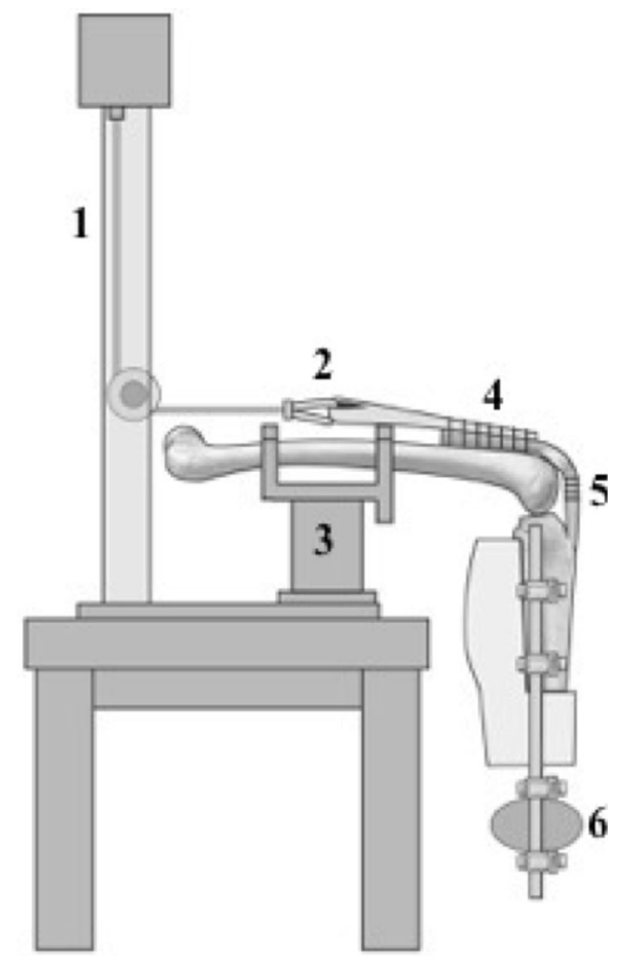

Fig. 5 The schematic diagram of the testing apparatus shows: (1) the cable-pulley mechanism attached to the Instron ${ }^{\mathbb{B}}$ testing machine; (2) the loop in the nylon webbing attached to the cable; (3) the custom femur mounting jig; (4) the quadriceps tendon attached to nylon webbing; (5) the patellar tendon repair site with reflective markers; and (6) 5-pound weight attached to the tibia $33-\mathrm{cm}$ distal to the knee axis of rotation. (Published with permission from Scott Bodell, Bodell Communications Inc, Dallas, TX, USA.)

be independent input variables to the resultant gap. The paired t-test was used for ascertaining the effects of repair technique on load-to-failure data. A p value less than 0.05 was considered significant. Correlation analyses were conducted using a Pearson's correlation after confirming for data normality and equal variance. The sample size powered the experiment to at least a value $(1-\beta)$ of 0.60 . All statistical calculations were performed using GraphPad Prism ${ }^{\circledR}$ (GraphPad Software, La Jolla, CA, USA).

\section{Results}

Mean gap formation was less in the augmentation group at all cycle counts $(\mathrm{p}<0.01)$ (Fig. 7). After the first 50 cycles, the mean gap was $271 \%$ greater in the control compared with the augmented repair group $(\mathrm{p}<0.01)$. At 250 cycles, a $68 \%$ increase in mean gap formation was seen for the control group (control: $5.96 \pm 0.86 \mathrm{~mm}$ [95\% CI, $5.30-6.62 \mathrm{~mm}$ ]; augmentation: $3.55 \pm 0.56 \mathrm{~mm}$ [95\% CI, 3.12-3.98 mm]; $p=0.02$ ). The age of the specimen did not affect gap formation for either the control or augmentation group, with the numbers available $(\mathrm{p}=0.63)$.

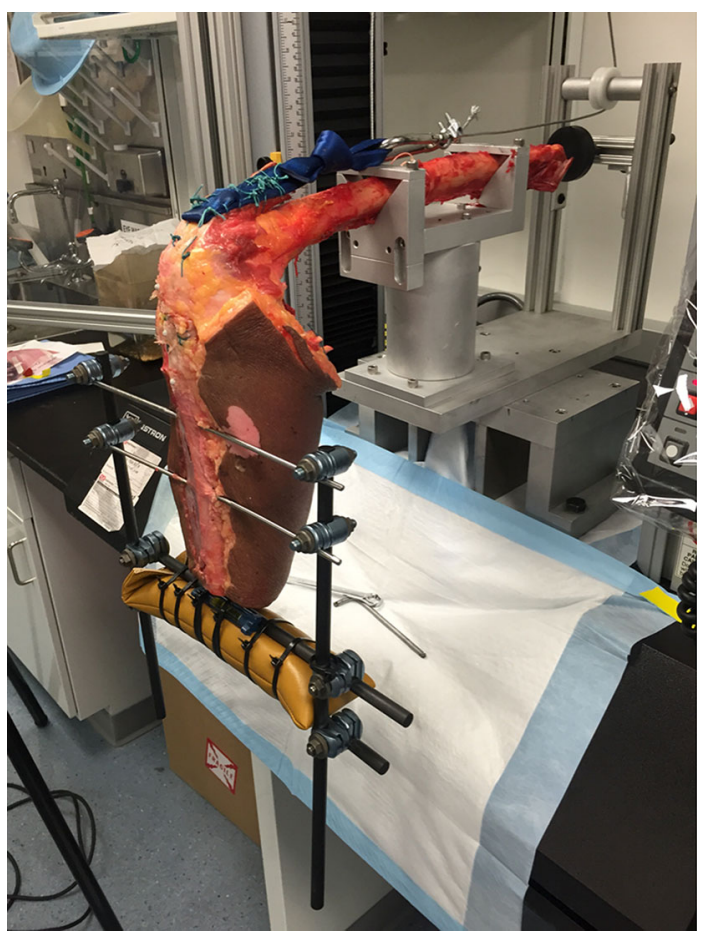

Fig. 6 A cadaveric specimen is shown mounted in the testing apparatus ready to proceed with cyclic load testing.

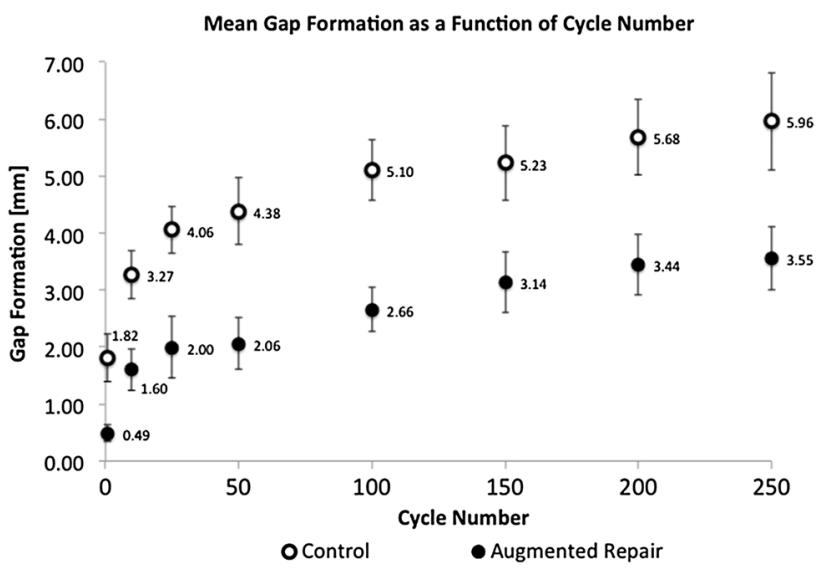

Fig. 7 The mean gap formation for the augmented repair group was lower than that of the control group for all cycles (ANOVA; $p<$ 0.01). Error bars $=\mathrm{SD}$ for each data point.

Maximum load to failure also showed increased strength for the augmentation group. The mean load to failure was 13\% greater in the augmentation group (control: $899.57 \pm$ $96.94 \mathrm{~N}$ [95\% CI, 825.06-974.09 N]; augmentation: $1030.70 \pm 122.41 \mathrm{~N}$ [95\% CI, 936.61-1124.79 N]; $\mathrm{p}=$ 0.01). All samples failed at the suture repair site with the exception of two that underwent the augmentation technique, which failed at the quadriceps tendon interface with the testing apparatus. With respect to failure load, age did not affect the biomechanical behavior of the patellar tendons, with the numbers available $(\mathrm{p}=0.17)$. 


\section{Discussion}

Prompt surgical repair of patellar tendon ruptures is preferred in patients fit for surgery to restore the extensor mechanism and allow optimal return to function $[22,28,37]$, but studies disagree about the best surgical technique [7, 11, 15, 25-28]. Despite routine use of the transosseous tunnel and modified-Krackow suture technique, little is known about the biomechanical properties of this repair method. Alternative fixation options such as suture anchors and various augmentation techniques have been described but supported with limited biomechanical evidence $[3,7,13,15,16,23,30,36,40]$. Our study provides a better understanding of the gap formation that can occur after patellar tendon repair and our proposed augmentation technique showed a substantial decrease in gap formation and an increase in ultimate strength of the repair compared with a common repair technique.

There are several limitations to this study. First, to our knowledge there are no studies defining patellar tendon repair failure, specifically evaluating critical gap formation and how much load is experienced across the repair site in clinical scenarios. Thus, it is difficult to translate these results in clinical practice. However, we do appreciate that gap and excessive load can result in patellar tendon repair failure, so surgeons historically have tried to mitigate the risk by adding augmentation to these repairs. For surgeons who are concerned for failure or previously have used augmentation, this technique can provide an alternative method for strengthening the repair. Second, our goal was to define a simple augmentation technique that decreases gap formation and increases repair strength, with minimal downside. However, we recognize that switching to a new technique comes with a learning curve and comfort factor. We believe this augmentation method is simpler than other previously described techniques [13, 15, 16, 23, 25, 30, 36] and does not require additional exposure or instrumentation. As with many new techniques, an initial attempt with the technique in a cadaver laboratory setting could prove beneficial but we believe the technique can be incorporated easily in clinical practice.

As with any biomechanical study, it can be difficult to translate the properties of cadaveric tissue and controlled biomechanical testing protocol to the complex scenarios imparted by in vivo situations. No data exist that define failure parameters in a clinical setting, which can make a direct comparison difficult; however, the protective benefits of our augmentation as shown in this laboratory study provide an impetus to explore the technique clinically. Additionally, our model for simulating a patellar tendon rupture by transecting the tendon off the inferior pole of the patella may not represent the commonly encountered longitudinally shredded appearance of the in vivo tendon rupture. In the standard transosseous tunnel repair and augmented repair, the primary method for securing the tendon involves the modified-Krackow stitch. The augmentation suture is independent of the tendon fixation, as it secures the modified-Krackow loops to the patella, therefore our technique should work regardless of the characteristics or location of the ruptured tendon assuming the modified-Krackow fixation is adequate. This same concept can be used to appreciate how the age and quality of cadaver tissue may not be important when directly comparing the difference between the control and augmentation techniques. Finally, although we were able to discern decreased gap formation and increased load to failure in the new technique, the sample size was relatively small, and the clinical relevance of the effect sizes we observed are unknown; however as there appears to be little disadvantage to this approach, the potential for a stronger repair-which may support earlier weightbearing and ROM-is appealing.

In the augmented and control groups, the majority of the gap formation occurred during the first 50 cycles. We speculate that the observed gap formation could result from the creep behavior of the tendon's response to the repair technique and suture cut-out of the tendon. The patellar tendon, like all viscoelastic materials, is susceptible to creep behavior where the tissues continue to deform under sustained load application. Moreover, creep can accumulate through repeated cycles. Although it is difficult to determine the cause of the initial gap formation, it appears consistent between the two testing groups.

In an attempt to avoid immobilization and encourage early weightbearing and knee motion, augmentation techniques have been proposed to provide additional strength to patellar tendon repairs. Despite the numerous small retrospective series on repair techniques with and without augmentation $[1,6,11,13,15,16,23,25,26,28,30,33$, $36,40]$, the biomechanical evidence is limited. In 2002, Ravalin et al. [35] were the first to describe systematic testing of the efficacy of suture and cable augmentation using a biomechanical model. Using 12 cadaver knees, they evaluated the repair site gap formation under cyclic loading for the common transosseous tunnel modifiedKrackow suture repair technique compared with two augmentation methods: a Number 5 Ethibond $^{\mathrm{TM}}$ or a $2.0-\mathrm{mm}$ Dall-Miles ${ }^{\circledR}$ cable (Stryker Corporation, Mahwah, NJ, USA) placed in a cerclage-loop fashion through the tibial tubercle and a horizontal patellar drill tunnel. Their reported gap formation across the standard suture repair averaged $7.3 \mathrm{~mm}$ while the Number 5 Ethibond $^{\mathrm{TM}}$ and Dall-Miles ${ }^{\circledR}$ cable augmentation groups averaged $4.9 \mathrm{~mm}$ and $3.5 \mathrm{~mm}$, respectively. They concluded that repair augmentation was necessary to decrease gap formation during early postoperative mobilization of the knee. The 
current study further supports the use of augmentation techniques, as our augmentation suture technique decreased gap formation during cyclic loading.

The biomechanical testing protocol described by Ravalin et al. [35] has been replicated to evaluate the efficacy of suture anchors as an alternative repair method for patellar tendon ruptures. In 2006, Bushnell et al. [3] compared transosseous suture repair using either Number 2 FiberWire ${ }^{\circledR}$ (Arthrex ${ }^{\circledR}$ Inc, Naples FL, USA) or Number 5 Ethibond $^{\mathrm{TM}}$ versus suture anchor repair with Number 2 FiberWire ${ }^{\circledR}$. Using six matched pairs of cadaveric knees, they reported a mean gap formation of $4.1 \mathrm{~mm}$ for suture anchor repair compared with $6.7 \mathrm{~mm}$ for the FiberWire ${ }^{\circledR}$ transosseous repair and $8.5 \mathrm{~mm}$ for the Ethibond ${ }^{\mathrm{TM}}$ group. They were the first to describe a ramp-up load to failure testing protocol to determine the maximum load sustained across the repair techniques. For suture anchors, the mean load to failure was $779 \mathrm{~N}$ compared with $730 \mathrm{~N}$ and $763 \mathrm{~N}$ for the Number 2 FiberWire $^{\circledR}$ and Number 5 Ethibond $^{\mathrm{TM}}$ transosseous repairs respectively. Despite having a decrease in gap formation with suture anchors, there was no difference in load to failure between the different techniques. In a similar study by Ettinger et al. [7], maximum load to failure for titanium and hydroxyapatite suture anchors was reported to be $597 \mathrm{~N}$ and $689 \mathrm{~N}$, respectively. For transosseous suture repair, the maximum load to failure was $301 \mathrm{~N}$. However, they performed a modified transosseous suture repair using Number 2 Ultrabraid $^{\mathrm{TM}}$ (Smith \& Nephew, Memphis, TN, USA) suture with the knots tied at the patellar tendon end, rather than over the superior pole of the patella. When comparing our load to failure with those of previous studies [3,7], we found that the mean load to failure for the standard transosseous suture technique was $899.57 \mathrm{~N}$ and the mean load to failure for the augmentation technique was $1030.7 \mathrm{~N}$, both of which are higher than previously reported. Variations in repair techniques and testing protocols could account for this difference, however our increased load to failure with the augmentation technique compared with our control shows a successful increase in the strength of the repair.

The findings from our study show that augmentation of patellar tendon repairs can decrease gap formation across the repair site and increase the overall strength of the repair. Our proposed augmentation technique performed extremely well in biomechanical testing, providing support for future clinical investigations comparing this method with others that are in common use, such as transosseous suture repair [27] and suture anchor fixation [3, 7]. Additionally, this study has highlighted our continued need to investigate what constitutes the definition and mode of failure of clinically significant patellar tendon repair failures, so we can further direct our research efforts.
Acknowledgments We thank the Washington University Musculoskeletal Research Core NIH P30 AR057235 (St Louis, MO, USA) for laboratory space, equipment and technical support, and Daniel Leib MS (Musculoskeletal Research Center, Washington University Department of Orthopaedics, St Louis, MO, USA) for providing technical laboratory assistance. We also thank Scott Bodell (Bodell Communications Inc, Dallas, TX, USA) for creation of medical illustrations.

\section{References}

1. Bhargava SP, Hynes MC, Dowell JK. Traumatic patella tendon rupture: early mobilisation following surgical repair. Injury. 2004;35:76-79.

2. Bonnin M, Lustig S, Huten D. Extensor tendon ruptures after total knee arthroplasty. Orthop Traumatol Surg Res. 2016;102(1 suppl):S21-31.

3. Bushnell BD, Byram IR, Weinhold PS, Creighton RA. The use of suture anchors in repair of the ruptured patellar tendon: a biomechanical study. Am J Sports Med. 2006;34:1492-1499.

4. Clayton RA, Court-Brown CM. The epidemiology of musculoskeletal tendinous and ligamentous injuries. Injury. 39:13381344.

5. Dempster WT. Free-body diagrams as an approach to the mechanics of human posture and motion. In Evans FG, ed. Biomechanical Studies of the Musculoskeletal System. Springfield, IL, Charles C Thomas; 1961:81-93.

6. Enad JG, Loomis LL. Primary patellar tendon repair and early mobilization: results in an active-duty population. J South Orthop Assoc. 2001;10:17-23.

7. Ettinger M, Dratzidis A, Hurschler C, Brand S, Calliess $T$, Krettek C, Jagodzinski M, Petri M. Biomechanical properties of suture anchor repair compared with transosseous sutures in patellar tendon ruptures: a cadaveric study. Am J Sports Med. 2013;41:2540-2544.

8. Galloway MT, Lalley AL, Shearn JT. The role of mechanical loading in tendon development, maintenance, injury, and repair. $J$ Bone Joint Surg Am. 2013;95:1620-1628.

9. Gelberman RH, Manske PR, Vande Berg JS, Lesker PA, Akeson WH. Flexor tendon repair in vitro: a comparative histologic study of the rabbit, chicken, dog, and monkey. J Orthop Res. 1984;2:39-48.

10. Gelberman RH, Menon J, Gonsalves M, Akeson WH. The effects of mobilization on the vascularization of healing flexor tendons in dogs. Clin Orthop Relat Res. 1980;153:283-289.

11. Greis PE, Holmstrom MC, Lahav A. Surgical treatment options for patella tendon rupture: Part I. Acute. Orthopedics. 2005;28:672-679; quiz 680-671.

12. Herbort M, Raschke M. [Ligament ruptures of the lower extremity in the elderly] [in German]. Unfallchirurg. 2011;114:671-680.

13. Hsu KY, Wang KC, Ho WP, Hsu RW. Traumatic patellar tendon ruptures: a follow-up study of primary repair and a neutralization wire. J Trauma. 1994;36:658-660.

14. Kannus P, Józsa L. Histopathological changes preceding spontaneous rupture of a tendon: a controlled study of 891 patients. $J$ Bone Joint Surg Am. 1991;73:1507-1525.

15. Kasten P, Schewe B, Maurer F, Gosling T, Krettek C, Weise K. Rupture of the patellar tendon: a review of 68 cases and a retrospective study of 29 ruptures comparing two methods of augmentation. Arch Orthop Trauma Surg. 2001;121:578-582.

16. Kinmont JC, Walter E, Curtis MJ. Augmentation of patellar tendon repair with poly-p-dioxannone cord. Injury. 2002;33:263264. 
17. Kovacev N, Antic J, Gvozdenovic N, Obradovic M, Vranjes M, Milankov M. Patellar tendon rupture: treatment results. Med Pregl. 2015;68:22-28.

18. Krackow KA, Thomas SC, Jones LC. A new stitch for ligamenttendon fixation: brief note. J Bone Joint Surg Am. 1986;68:764766.

19. Krushinski EM, Parks BG, Hinton RY. Gap formation in transpatellar patellar tendon repair: pretensioning Krackow sutures versus standard repair in a cadaver model. Am J Sports Med. 2010;38:171-175.

20. Kuechle DK, Stuart MJ. Isolated rupture of the patellar tendon in athletes. Am J Sports Med. 1994;22:692-695.

21. Laros GS, Tipton CM, Cooper RR. Influence of physical activity on ligament insertions in the knees of dogs. J Bone Joint Surg Am. 1971;53:275-286.

22. Larsen E, Lund PM. Ruptures of the extensor mechanism of the knee joint: clinical results and patellofemoral articulation. Clin Orthop Relat Res. 1986;213:150-153.

23. Larson RV, Simonian PT. Semitendinosus augmentation of acute patellar tendon repair with immediate mobilization. Am J Sports Med. 1995;23:82-86.

24. Lehto M, Duance VC, Restall D. Collagen and fibronectin in a healing skeletal muscle injury: an immunohistological study of the effects of physical activity on the repair of injured gastrocnemius muscle in the rat. J Bone Joint Surg Br. 1985;67:820-828.

25. Levy M, Goldstein J, Rosner M. A method of repair for quadriceps tendon or patellar ligament (tendon) ruptures without cast immobilization: preliminary report. Clin Orthop Relat Res. 1987;218:297-301.

26. Lindy PB, Boynton MD, Fadale PD. Repair of patellar tendon disruptions without hardware. J Orthop Trauma. 1995;9:238243.

27. Marder RA, Timmerman LA. Primary repair of patellar tendon rupture without augmentation. Am J Sports Med. 1999;27:304307.

28. Matava MJ. Patellar tendon ruptures. J Am Acad Orthop Surg. 1996;4:287-296.
29. McMaster PE. Tendon and muscle ruptures: clinical and experimental studies on the causes and location of subcutaneous ruptures. J Bone Joint Surg Am. 1933;15:705-722.

30. Miskew DB, Pearson RL, Pankovich AM. Mersilene strip suture in repair of disruptions of the quadriceps and patellar tendons. $J$ Trauma. 1980;20:867-872.

31. Noyes FR, Torvik PJ, Hyde WB, DeLucas JL. Biomechanics of ligament failure: II. An analysis of immobilization, exercise, and reconditioning effects in primates. J Bone Joint Surg Am. 1974;56:1406-1418.

32. Ong BC, Sherman O. Acute patellar tendon rupture: a new surgical technique. Arthroscopy. 2000;16:869-870.

33. Ramseier LE, Werner CM, Heinzelmann M. Quadriceps and patellar tendon rupture. Injury. 2006;37:516-519.

34. Rand JA, Morrey BF, Bryan RS. Patellar tendon rupture after total knee arthroplasty. Clin Orthop Relat Res. 1989;244:233238.

35. Ravalin RV, Mazzocca AD, Grady-Benson JC, Nissen CW, Adams DJ. Biomechanical comparison of patellar tendon repairs in a cadaver model: an evaluation of gap formation at the repair site with cyclic loading. Am J Sports Med. 2002;30:469-473.

36. Shelbourne KD, Darmelio MP, Klootwyk TE. Patellar tendon rupture repair using Dall-Miles cable. Am J Knee Surg. 2001;14:17-20; discussion 20-21.

37. Siwek CW, Rao JP. Ruptures of the extensor mechanism of the knee joint. J Bone Joint Surg Am. 1981;63:932-937.

38. Taylor DC, Dalton JD Jr, Seaber AV, Garrett WE Jr. Viscoelastic properties of muscle-tendon units: the biomechanical effects of stretching. Am J Sports Med. 1990;18:300-309.

39. Webb LX, Toby EB. Bilateral rupture of the patella tendon in an otherwise healthy male patient following minor trauma. $J$ Trauma. 1986;26:1045-1048.

40. West JL, Keene JS, Kaplan LD. Early motion after quadriceps and patellar tendon repairs: outcomes with single-suture augmentation. Am J Sports Med. 2008;36:316-323. 